Revista ELectrónica de Investigación y EValuación Educativa

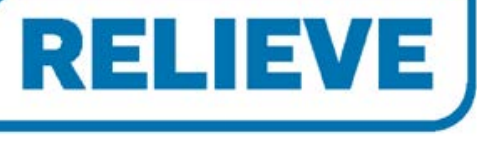

ISSN: 1134-4032
e-Journal of Educational Research, Assessment and Evaluation

\title{
Actitudes hacia personas refugiadas y ciudadanía europea inclusiva. Análisis para una propuesta educativa intercultural con el profesorado en formación
}

\section{Attitudes toward refugees and inclusive European citizenship. Analysis for an intercultural educational proposal with teachers in training}

Cala, Verónica C.; Soriano-Ayala, Encarnación \& López-Martínez Manuel J. Universidad de Almería.

\begin{abstract}
The recent migratory crisis has multiplied the number of school age people that are forced to immigrate. The integration of the minors in host countries demands not only schooling, but also an inclination of the educational community to accept the said reality and critically manage European immigration policies. At the European level there is a growing rejection toward the migratory phenomenon and European policies. This study analyzes the attitudes toward refugees through the perception of real threat, symbol, and the affective reactions that the future professors experience. Furthermore, it explores in what extent the attitudes toward refugees are related to an inclusive European citizenship. It involves survey research (a sample of 624 education students from southeast Spain in recent years) with a transversal design. The used instrument is a modified version of the scale of attitudes and a scale of inclusive European citizenship itself. The results show a marked indifference toward the related issues with refugees, but not a perceived elevated threat or marked emotional reactions. While the attitudes toward refugees are not very positive, there is a positive inclusive European citizenship that legitimizes the reception of refugees in European states, the cession of equal rights and possibilities of new forms of coexistence. This study manifests a marked correlation between attitudes toward refugees and inclusive European citizenship, where the perceived threat appears like the principal predictor of inclusive citizenship. The results permit us to place these future teachers inside the European panorama as one of the social sectors less affected by the growing xenophobia and discredit toward the European Union. However, they are precise and urgent programs of training in intercultural, antiracist and migratory education
\end{abstract}

Keywords: Attitudes towards refugees; intercultural; faculty; European intercultural citizenship

\section{Resumen}

La reciente crisis migratoria ha multiplicado el número de personas en edad escolar que migran forzosamente. La integración de los menores en los países de acogida exige no solo su escolarización sino una inclinación de la comunidad educativa para aceptar dicha realidad y gestionar críticamente las políticas europeas migratorias. A nivel europeo hay un creciente rechazo hacia el fenómeno migratorio y hacia las políticas europeas. El estudio analiza las actitudes hacia los refugiados mediante la percepción de amenaza real y simbólica y las reacciones afectivas que los futuros profesores experimentan. Además, explora en qué medida las actitudes hacia los refugiados se relacionan con una ciudadanía europea inclusiva. Se trata de una investigación mediante encuesta (una muestra de 624 estudiantes de los últimos años de magisterio del sureste español) con un diseño transversal. El instrumento utilizado es una versión modificada de las escalas de actitudes
Reception Date 2018 September 18

Approval Date 2018 December 07

Publication Date: 2018 December 10
Fecha de recepción 2018 Septiembre 18

Fecha de aprobación 2018 Diciembre 07

Fecha de publicación 2018 Diciembre 10 


\begin{abstract}
de Amenaza realista y simbólica, Escala de actitudes prejuiciosas, y una Escala de Ciudadanía Europea Inclusiva de elaboración propia. Los resultados muestran una marcada indiferencia hacia las cuestiones relacionadas con las personas refugiadas, no existiendo una amenaza percibida elevada ni reacciones emocionales marcadas. Si bien las actitudes hacia refugiados manifiestan desinterés, sí existe una ciudadanía europea inclusiva positiva que legitima la acogida de refugiados de los estados europeos, la cesión de iguales derechos y posibilidades de nuevas formas de convivencia. El estudio manifiesta una marcada correlación entre las actitudes hacia los refugiados y la ciudadanía europea inclusiva, donde una menor amenaza percibida aparece como principal predictor de una mayor ciudadanía inclusiva. Los resultados nos permiten situar a estos futuros docentes dentro del panorama europeo como uno de los sectores sociales menos afectados por la creciente xenofobia y descrédito hacia la Unión Europea. No obstante, son precisos y urgentes programas de formación en educación intercultural, antirracista y de atención a los refugiados.
\end{abstract}

Palabras clave: Actitudes hacia refugiados; profesorado; ciudadanía europea intercultural

En 2017 el número de desplazados forzosos aumentó a 68,5 millones de personas, de los cuales un $52 \%$ son menores de 18 años. En los últimos diez años la cifra de personas que migran contra su voluntad ha aumentado más del 50\%. De modo que, en la actualidad, 1 de cada 110 personas del mundo está desplazada y 1 de cada 220 es menor de edad (ACNUR, 2018). En muchos casos el desplazamiento se produce en condiciones de extrema peligrosidad. La Organización Internacional para las Migraciones estima que en 2017 llegaron a Europa cerca de 186 mil personas migrantes a través de rutas mediterráneas (OIM, 2017). Estas cifras suponen, para muchos, la crisis humanitaria más importante después de la Segunda Guerra Mundial.

Las organizaciones internacionales consideran una prioridad atender la cuestión migratoria internacional desde el ámbito socioeducativo, acogiendo a menores $\mathrm{y}$ adultos en los sistemas educativos para favorecer su subsistencia e integración en los países de acogida (ACNUR, 2018; 2016; Wrench et al. 2018). No obstante, la atención educativa requiere una adaptación de los propios sistemas considerando las problemáticas específicas asociadas a los niños y jóvenes refugiados como son las dificultades en el acceso a la escolarización, la incomprensión del proceso migratorio por parte del profesorado y compañeros que a veces no son conscientes del trauma causado por el conflicto bélico en el niño refugiado, falta de sentimiento de pertenencia, barreras idiomáticas, segregación y discriminación en entornos escolares (Dryden-Peterson, 2011, 2016). Frente a esas problemáticas, es preciso desarrollar nuevas estrategias educativas que respondan a los fenómenos migratorios globales (Banks, Suárez-Orozco, \& BenPeretz, 2016), sabiendo que el desarrollo de políticas y programas educativos inclusivos e interculturales permite mejorar las actitudes hacia las personas migrantes y refugiadas y una disminución de la xenofobia escolar (Sharkey, 2018). Las actitudes hacia las personas refugiadas aparecen como una cuestión central en el desarrollo de políticas educativas interculturales. Tomkinson (2018) muestra cómo las creencias y actitudes hacia los refugiados cumplen una función importante en la gestión de la migración.

El papel del profesorado aparece como una de las claves en el proceso de recepción e integración de los refugiados. Las investigaciones realizadas en EEUU reflejan cómo, a pesar de su larga historia receptora de refugiados, el profesorado sigue sin estar preparado para ello, mostrando pocos conocimientos sobre la situación, actitudes negativas hacia los refugiados y baja sensibilidad y competencia intercultural ( $\mathrm{Li}$, 2018; McWilliams, 2015). El estudio de Tarman y Gürel (2017) realizado en Turquía con futuros maestros muestra un creciente desinterés por la cuestión migratoria y arroja peores actitudes entre aquellos que se encuentran en contextos con alto porcentaje 
de población refugiada. Asimismo, un estudio europeo multisituado con universitarios muestra que el imaginario hacia los refugiados que llegan a la Unión Europea se está asentando en estereotipos negativos y en discursos caracterizados por el miedo al otro. También alertan del desconocimiento y la desinformación reinante entre los estudiantes en tal materia (Padilla \& Goldberg, 2017).

\section{Actitudes hacia los refugiados}

La formación de actitudes no es una cuestión meramente individual ya que depende de una combinación compleja de circunstancias personales, valores y del entorno externo (discurso de políticos, políticas migratorias y sociales, medios de comunicación y sociedad civil). Las actitudes son relativamente estables como para ser medibles, pero son también sensibles a los cambios, pudiendo apreciar diferencias incluso entre regiones dentro de un mismo país (Drinkwater, Drinkwater, \& Kauser, 2013). Se pueden definir como el conjunto relativamente duradero de creencias, sentimientos y tendencias de comportamiento hacia objetos, grupos, eventos o símbolos socialmente significativos (Hogg 2006), en este caso hacia las personas refugiadas, es decir, aquellas personas que llevan a cabo una migración forzosa motivada por causas ajenas a su voluntad.

Los estudios sobre actitudes hacia refugiados se han ido diferenciando de aquellos centrados en personas migrantes al observarse que se encuentran condicionados por factores distintos. En el caso de las actitudes hacia refugiados existe una mayor influencia de factores político-ideológicos, como es la existencia de discursos xenófobos y de odio, mientras que hacia los refugiados tienen un mayor peso los factores económicos (Getmansky \& Zeitzoff, 2018; Steele \& Abdelaaty, 2018). Concretamente las actitudes hacia los refugiados se han relacionado con la identidad étnica y nacional, la cohesión social y la seguridad nacional, al tiempo que las preocupaciones relacionadas con los ingresos y la ocupación laboral (competencia en el mercado de trabajo) influyen en mayor medida en la actitud hacia los inmigrantes (Semyonov, Raijman \& Gorodzeisky, 2008).

\section{Teorías psicológicas sobre actitudes}

Desde la psicología social se han ido definiendo distintas teorías que explican la construcción de actitudes entre grupos humanos. Entre ellas destacan la teoría de la identidad social de Tajfel y Turner (1979) que analiza cómo el sentido de pertenencia a un grupo social motiva el comportamiento concreto hacia su propio grupo, y otro distinto hacia los que entiende como grupos distintos. Las identidades nacionales fuertes (defensoras de la seguridad nacional, de la cultura y valores tradicionales) se relacionan con mayor distancia, percepción de amenaza y rechazo hacia migrantes y refugiados que las identidades híbridas, cosmopolitas o interculturales (Dixon et al., 2018). La teoría del contacto de Allport y Pettigrew (Allport, 1954; Pettigrew, 1979,1998) argumenta que existen más prejuicios y distancia entre grupos cuando existe poca interacción entre los mismos. Y la teoría de la dominancia social de Sidanius y Pratto (1999) explica cómo se organizan las relaciones sociales en base a estructuras preexistentes como son el racismo, el sexismo o el nacionalismo, y cómo de acuerdo a esos sistemas de estratificación se constituyen las actitudes, valores y creencias entre los grupos. Los estudios que profundizan en los colectivos que solicitan asilo demuestran que existen distintos grados de amenaza según su procedencia, su racialización y su género, siendo más intenso el rechazo hacia colectivos musulmanes y procedentes de África (Yitmen \& Ve, 2017).

Por último, la que fundamenta este estudio, es la teoría integrada de la amenaza de Stephan \& Stephan (2000) que postula que un grupo desarrolla actitudes hostiles cuando sus miembros perciben en peligro su estatus colectivo empeorando las actitudes y el comportamiento hacia el exogrupo (Stephan \& Stephan, 1985; Stephan \& Renfro, 2002). De modo que, cuanto mayor es la amenaza sentida de un endogrupo (grupo social que se 
identifica en base a una serie de semejanzas) y cuanto más se cree que peligran sus recursos materiales (empleo, servicios sociales) y/o simbólicos (cultura, creencias), peores actitudes se manifiestan hacia el /los exogrupos (grupos sociales que son identificados como diferentes). La relación entre el endogrupo y el exogrupo se establece al configurarse un "nosotros" y un "ellos", habitualmente en términos dicotómicos. La identidad nacional es uno de los atributos más sólidos de conformación de semejanzas y sentimiento de pertenencia dentro de una comunidad, endogrupo, por lo que esta teoría ha sido ampliamente utilizada para analizar las relaciones entre autóctonos y personas que inmigran voluntaria o forzosamente. En la teoría integrada de la amenaza la percepción de amenaza opera independientemente de si la amenaza es real o tan solo sentida. El grado de amenaza percibido se ha relacionado con diversos factores como son el tamaño y condicionantes sociales del exogrupo y el endogrupo, la existencia de una ideología multiculturalista o el desarrollo de políticas de integración (Stark, Mäs \& Flache, 2015).

\section{Actitudes y ciudadanía europea inclusiva}

La gestión migratoria por parte de las instituciones europeas y las actitudes positivas para la acogida y la integración de personas refugiadas convergen en la noción de ciudadanía europea democrática e inclusiva (Gualda, 2011). El concepto de ciudadanía europea inclusiva (De Lucas, 2001) comprende una expansión del término de ciudadanía más allá de los límites de las nacionalidades de los estados miembros. Recordemos que la ciudadanía ha ido ligada a la noción de estado-nación. Esta expansión en el concepto de ciudadanía presume que los flujos migratorios exigen el reconocimiento del derecho universal a la libre circulación y la expansión del estatuto jurídico de ciudadano a todas las personas migrantes. Esto implica a su vez el reconocimiento de las identidades de las personas y los pueblos que llegan a Europa, en el respeto a las diferencias culturales $\mathrm{y}$, fundamentalmente, en la expansión de las libertades, derechos sociales y políticos a las personas inmigradas.
Como sostiene la teoría de la identidad social, la visión hacia las personas inmigradas y refugiadas se relaciona con la noción de ciudadanía que se construya. Según la construcción identitaria que exista, si integra o no en su definición al otro: refugiado y migrante, así variará la predisposición hacia las políticas de acogida y a modelos interculturales. Por ese motivo, el principal objetivo de este estudio es identificar las actitudes de los futuros maestros hacia los refugiados y su relación con el grado de ciudadanía europea inclusiva referida.

\section{Método}

\section{Participantes}

Los participantes fueron estudiantes universitarios de los grados de Educación Infantil y Primaria. Fueron seleccionados estos grados por tratarse de personas que, en un futuro cercano, potencialmente, enseñarán y serán modelos de niños y niñas, e incidirán sobre la percepción de la sociedad y la convivencia que en ella se establezca. De ellos, 150 (23,8\%) son hombres y 479 $(76,2 \%)$ mujeres. La edad media de la muestra ha sido de 21,78 años (22,36 de media los hombres y 21,55 las mujeres), con edades comprendidas entre los 18 y los 55 años.

\section{Instrumentos}

Junto a los datos personales, se recoge información de tres escalas, dos seleccionadas de otros estudios llevados a cabo con personas refugiadas e inmigradas y una tercera, de elaboración propia, centrada en la llegada, reconocimiento y acogida a los refugiados, especialmente de origen sirio, en la Unión Europea.

La primera escala la hemos denominado de Amenazas y Derechos y está basada en la Escala de Amenaza realista y simbólica (Realistic and symbolic threat scale) de Stepham, Ybarra y Bachman (1999). En nuestro caso hemos utilizado 9 de los 12 enunciados que la conforman. El análisis factorial realizado a los ítems seleccionados nos los clasifica en 2 factores, a los que hemos denominado subescala de Amenaza, 
compuesta por 6 enunciados (p.e. la acogida de refugiados está minando la cultura española), y la subescala de Derechos que contempla 3 enunciados (p.e. los refugiados tienen el mismo derecho a ayudas que los españoles en situación de pobreza). El formato de respuesta es una escala Likert de cinco puntos, que va de 1 "totalmente en desacuerdo" a 5 "totalmente de acuerdo". Todos los enunciados negativos se codifican de manera inversa y luego se promedian con los positivos, de modo que valores altos en esta escala determinan menos amenaza y más derechos de los recién llegados. La fiabilidad total de la escala ha sido de 0.755. La subescala de Amenaza arroja un $\alpha=0.754$ y la de Derechos $\alpha=0.64$.

La segunda escala la hemos nombrado de Reacciones afectivas. Esta escala está adaptada del cuestionario de Actitudes prejuiciosas (Prejudicial attitude survey) de Stephan y Stephan (1993) y de la escala que Garcia et al (2003) denominan Escala de Emociones. La escala original consta de 12 términos, 6 emociones (p.e. Odio) y 6 evaluativos (p.e. Admiración). Nosotros hemos modificado la escala y hemos añadido un término más quedando una escala de 13 términos (Simpatía, Odio, Admiración, Indiferencia, Confianza, Hostilidad, Rechazo, Aceptación, Afecto, Superioridad, Calidez, Disgusto y Vergüenza). Las respuestas fueron codificadas del 1 al 10, en las que se les pide a los estudiantes que indiquen la intensidad que le produce al pensar y ver a una persona refugiada (en concreto, los refugiados que llegan huyendo de las guerras a España y a la Unión Europea), siendo 1 nada y 10 mucho. Todas las palabras de valencia negativa (p.e Odio) se codifican de manera inversa y luego se promedian con las palabras de valencia positiva (p.e. Simpatía) de modo que los valores más altos reflejan menos sentimientos prejuiciosos, es decir, implica más aceptación y mayor afectividad positiva. Para nuestro estudio hemos utilizado las puntuaciones de la escala total y de la escala exclusiva de reacciones positivas. La escala total tiene una fiabilidad de 0.727 y la escala de reacciones positivas arroja un $\alpha=0.889$.
Por último, una escala de Ciudadanía europea inclusiva centrada en la percepción de la actuación de la Unión Europea y en las creencias ciudadanas acerca de la llegada de refugiados. Esta escala ha estado formada por once ítems, 3 con enunciados positivos (p.e. la llegada de las personas refugiadas a la Unión Europea es una oportunidad para educar en la convivencia pacífica) y 8 enunciados negativos (p.e. la crisis de los refugiados puede romper la Unión Europa). El formato de respuesta es una escala Likert de 5 puntos que va de 1 totalmente en desacuerdo a 5 puntos totalmente de acuerdo. Como en las otras dos escalas, todos los enunciados negativos se codifican de manera inversa y luego se promedian con los enunciados formulados positivamente, donde la puntuación más alta muestra mayor acogimiento y visión positiva hacia los refugiados que llegan a la Unión Europea. Para esta escala la fiabilidad es de 0.703 .

\section{Procedimiento}

Los participantes fueron invitados a participar voluntariamente, y se les informó que el estudio exploraría las actitudes hacia las personas refugiadas garantizando el anonimato. Una vez proporcionado el consentimiento informado, los participantes procedieron a completar los datos personales, tres ítems sobre ciudadanía y las tres escalas que componen el instrumento de recogida de datos. Las investigadoras lo aplicaron en las aulas con la presencia del profesor de la asignatura, y se pusieron a disposición de los estudiantes para solucionar todas las dudas que pudieran aparecer.

\section{Resultados}

\section{Estadística descriptiva}

Las escalas de Amenaza y Derechos, la de Reacciones afectivas y la escala de Ciudadanía europea inclusiva no tienen puntos de corte cuantitativos para descifrar actitudes y prejuicios, bajas, moderadas y altas. Por lo tanto, se realizaron estadísticas descriptivas (Media y Desviación Estándar) para cada una de las tres pruebas. 
Tabla 1: Estadística descriptiva de las escalas y correlación entre ellas

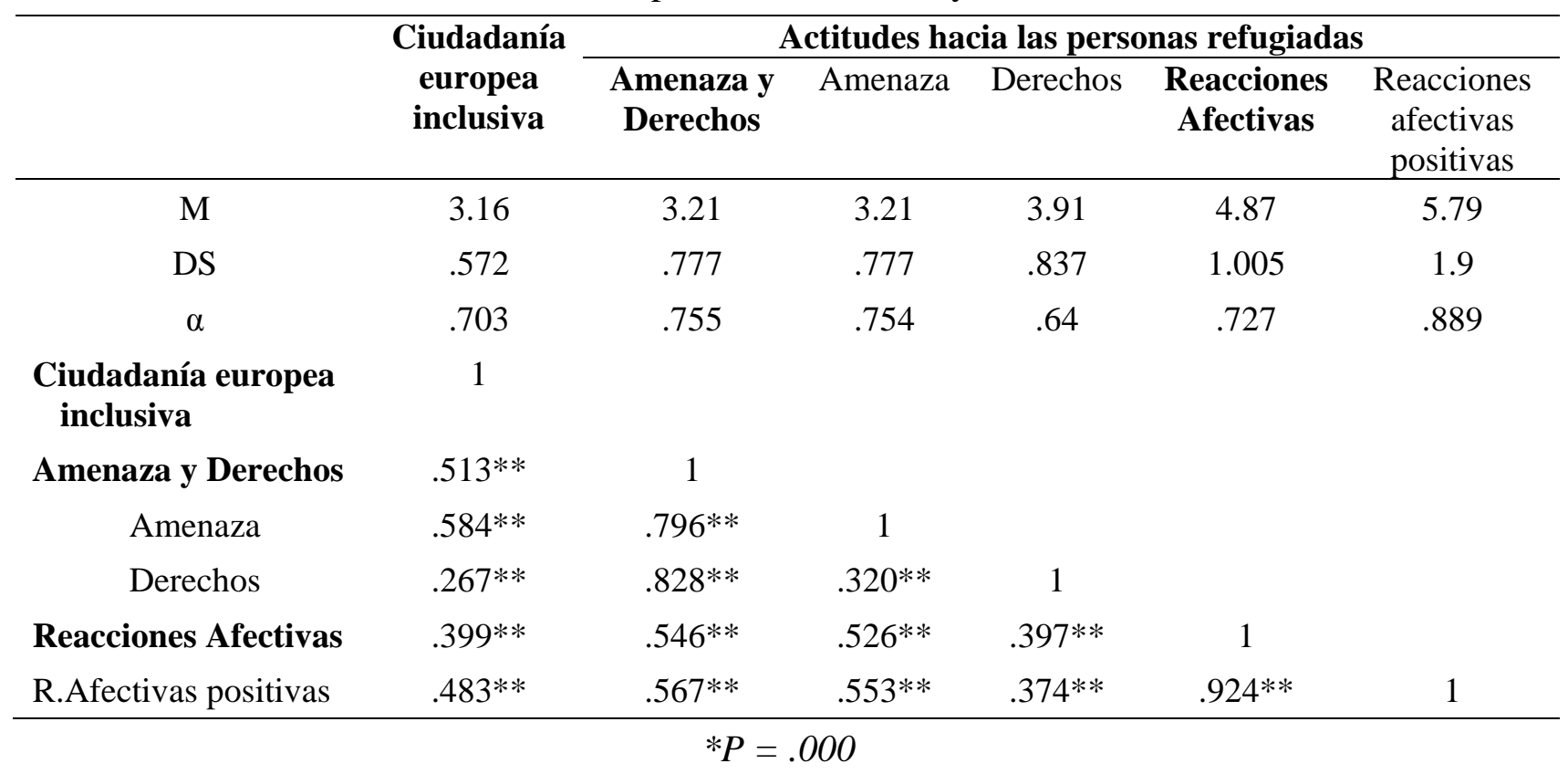

La puntuación media más alta en las escalas que puntúan del 1 al 5 se obtiene en la escala de derechos (3.91). El resto de las puntuaciones están próximas a 3 por el límite superior lo que se puede interpretar como cierta indiferencia o mínimo de actitud positiva por parte de los participantes hacía las personas refugiadas. Por otra parte, la escalas que puntuaban del 1 al 10 , la de Reacciones afectivas, presenta una media que no llega a 5 y en Reacciones afectivas positivas se alcanza una puntuación de 5.79, mostrando también actitud neutra.

\section{Correlaciones entre las escalas y estudio predictivo}

Se observan correlaciones positivas entre todas las escalas. En la Ciudadanía europea inclusiva, la correlación más alta (.584) se obtiene con la subescala de Amenaza, seguida de la escala total Amenaza y Derechos (.513), $\mathrm{y}$ de la subescala de reacciones afectivas positivas (.483). La subescala de reacciones afectivas positivas y la escala de reacciones afectivas correlacionan con la subescala de Amenaza (.553 y .526 respectivamente).

Para conocer cómo se comporta la variable criterio ciudadanía europea inclusiva, considerando la puntuación obtenida en este cuestionario, en función de las variables independientes o predictores "subescalas de Derechos, Reacciones afectivas positivas y Amenaza”, realizamos una regresión jerárquica.

Tabla 2: Regresión jerárquica para la Ciudadanía europea inclusiva

\begin{tabular}{lcccc}
\hline & $\mathrm{R}^{2}{ }_{\mathrm{c}}$ & $\beta$ & $\mathrm{t}$ & $\mathrm{p}$ \\
\hline $\begin{array}{l}\text { Modelo 1 } \\
\quad .332\end{array}$ & & .578 & 16.36 & .000 \\
$\quad$ Amenaza & & & & \\
Modelo 2 & .367 & & & \\
$\quad$ Amenaza & & .444 & 10.553 & .000 \\
$\quad$ Reacciones afectivas positivas & & .232 & 5.53 & .000 \\
\hline
\end{tabular}


A través de pasos sucesivos se reconocen 2 modelos, el sistema rechaza un tercer modelo en el que se introduce como regresor la puntuación de la subescala derechos. Se puede decir que no es un regresor que aporte nada sustantivo.

El primer modelo explica 33,2\%, la prueba de análisis de la varianza nos muestra la varianza explicada por la regresión, en donde el valor de $\mathrm{F}$ es 267,74 cuya probabilidad asociada es .000 , lo que nos indica un efecto real de la variable Amenaza sobre la variable criterio "Ciudadanía europea inclusiva" que recoge también creencias ciudadanas ante la llegada de refugiados.

El segundo modelo explica el 36,7\%. El valor de $\mathrm{F}$ es 156,56 y la probabilidad asociada es .000, lo que muestra un efecto real de las variables Amenaza y Reacciones afectivas positivas sobre la variable criterio.

La correlación que hemos visto entre los dos predictores podría sugerir un problema de multicolinealidad. El factor de inflación de la varianza (VIF) fue inferior a 1.5 y el índice de condición inferior a 10, por lo que se descarta la multicolinealidad.

En ambos modelos la actitud prejuiciosa de Amenaza percibida es la que tiene mayor peso específico. En el segundo modelo el peso de las reacciones afectivas positivas es la mitad que la Amenaza percibida.

Lo que explican estos modelos es que, en nuestros futuros maestros, la actitud de aceptación y acogimiento hacia los refugiados, sobre todo de origen sirio que llegan a la Unión Europea, depende de la amenaza percibida, y tienen menos peso las reacciones positivas que les pueda producir estas personas desprotegidas que piden refugio en la Unión Europea. En cambio, los conocimientos y la forma de pronunciarse de los estudiantes sobre los derechos que poseen los refugiados, no inciden ni en la aceptación ni en el acogimiento de los mismos.

\section{Estudio comparativo del percentil menor y mayor de 50 en la escala de ciudadanía europea inclusiva}

La forma de responder de los futuros maestros a las escalas y, en concreto, a la escala de Ciudadanía europea inclusiva con puntuaciones la mayoría próximas a 3 , ya sean por el límite superior como por el inferior, nos ha invitado a conocer cómo responden los jóvenes que se encuentran en el percentil $50 \mathrm{y}$ los que se encuentran en el superior. La media de los jóvenes situados en el percentil 50 es de $\mathrm{M}=2,94$ (DS= .51) y los jóvenes situados en el superior $\mathrm{M}=3.43$ (DS=.51), observamos que la actitud de ambos se aproxima a 3 ; es decir, que manifiestan cierta indiferencia, unos con una actitud suavemente negativa, y los otros ligeramente positiva, aunque sí se observan diferencias significativas al realizar la t entre ambas puntuaciones $(\mathrm{t}=-11.4 \mathrm{y} \mathrm{p}=.000)$.

\section{Análisis de diferencias por género}

Las respuestas a los ítems que reflejan percepciones y creencias de los estudiantes hacia los refugiados en la Unión Europea, que incluyen los ítems de la escala de Ciudadanía europea inclusiva más otros tres relacionados (1. La llegada de los refugiados es un examen a la UE para comprobar si defienden los derechos humanos, 2. La crisis de los refugiados es un problema político más que humanitario, y 3. La actuación de la UE con los refugiados no favorece la integración social de estas personas) también suelen mostrar puntuaciones próximas a 3, y excepto en el segundo ítem que la diferencia es significativa y la $r$ de Rosenthal muy pequeña, en el resto las respuestas tanto de hombres como de mujeres son similares. 
Cala, Verónica C.; Soriano-Ayala, Encarnación \& López-Martínez Manuel J. (2018). Actitudes hacia personas refugiadas y ciudadanía europea inclusiva. Análisis para una propuesta educativa intercultural con el profesorado en formación. RELIEVE, 24(2), art. M1. doi: http://doi.org/10.7203/relieve.24.2.13320

Tabla 3: Diferencias de género en los ítems de Ciudadanía europea inclusiva

\begin{tabular}{|c|c|c|c|c|c|c|c|c|}
\hline & \multicolumn{2}{|c|}{ Hombres } & \multicolumn{2}{|c|}{ Mujeres } & \multirow[b]{2}{*}{$\mathrm{U}$} & \multirow[b]{2}{*}{$\mathrm{Z}$} & \multirow[b]{2}{*}{$\mathrm{p}$} & \multirow[b]{2}{*}{$r$} \\
\hline & $\mathrm{M}$ & DS & $\mathrm{M}$ & DS & & & & \\
\hline $\begin{array}{l}\text { Considero a las personas refugiadas en la UE } \\
\text { como ciudadanos/as con plenos derechos }\end{array}$ & 3.91 & 1.09 & 4.01 & 1.04 & 33757.5 & -.938 & .348 & \\
\hline $\begin{array}{l}\text { La llegada de los refugiados desde } 2015 \text { es un } \\
\text { examen a la UE para comprobar si defiende los } \\
\text { Derechos Humanos }\end{array}$ & 3.41 & 1.28 & 3.14 & 1.26 & 30569.5 & -2.390 & .017 & -.09 \\
\hline $\begin{array}{l}\text { La democracia europea ha sido afectada } \\
\text { negativamente por la llegada de personas } \\
\text { refugiadas a su territorio }\end{array}$ & 2.66 & 1.27 & 2.56 & 1.10 & 33956 & -.652 & .514 & \\
\hline $\begin{array}{l}\text { El aprecio hacia la diversidad cultural y étnica } \\
\text { en la ciudadanía europea ha disminuido con la } \\
\text { llegada de personas refugiadas }\end{array}$ & 3 & 1.16 & 2.85 & 1.17 & 32912.5 & -1.205 & .228 & \\
\hline $\begin{array}{l}\text { La llegada de personas refugiadas a la UE puede } \\
\text { ser una oportunidad para educar en la } \\
\text { convivencia pacífica }\end{array}$ & 3.87 & 1.01 & 3.88 & 1.1 & 333305 & -.62 & .536 & \\
\hline $\begin{array}{l}\text { La crisis de los refugiados es un problema } \\
\text { político más que humanitario }\end{array}$ & 3.46 & 1.26 & 3.55 & 1.16 & 34287 & -.554 & .538 & \\
\hline La crisis de los refugiados puede romper la UE & 2.29 & 1.1 & 2.40 & 1.14 & 32769.5 & -1.05 & .293 & \\
\hline $\begin{array}{l}\text { La crisis de los refugiados puede deteriorar el } \\
\text { consenso sobre los valores democráticos que } \\
\text { sustenta la UE }\end{array}$ & 2.88 & 1.14 & 2.80 & 1.13 & 33611.5 & -.637 & .524 & \\
\hline $\begin{array}{l}\text { La llegada de los refugiados a la UE ha } \\
\text { provocado que aumente el rechazo de la } \\
\text { ciudadanía europea hacia estas personas }\end{array}$ & 3.37 & 1.13 & 3.29 & 1.17 & 33980.5 & -.678 & .498 & \\
\hline $\begin{array}{l}\text { La llegada de los refugiados a la UE ha } \\
\text { reflejado la falta de consenso entre los } \\
\text { dirigentes de las naciones }\end{array}$ & 3.75 & 1.03 & 3.60 & .99 & 32062 & -1.893 & .058 & \\
\hline $\begin{array}{l}\text { La crisis de los refugiados en la UE es un } \\
\text { problema sólo humanitario }\end{array}$ & 1.93 & .96 & 2.08 & 1.05 & 32500 & -1.426 & .154 & \\
\hline $\begin{array}{l}\text { La actuación de la UE con los refugiados no } \\
\text { favorece la integración social de estas personas }\end{array}$ & 3.60 & 1.07 & 3.55 & 1.06 & 33795 & -.66 & .51 & \\
\hline $\begin{array}{l}\text { La UE ha actuado acertadamente para evitar la } \\
\text { entrada masiva de personas refugiadas porque } \\
\text { entre ellos se encuentran terroristas }\end{array}$ & 2.5 & 1.24 & 2.63 & 1.13 & 32782.5 & -1.224 & .221 & \\
\hline $\begin{array}{l}\text { Debido a la crisis económica no hay suficiente } \\
\text { sitio en la UE para acoger a las personas } \\
\text { refugiadas }\end{array}$ & 2.72 & 1.36 & 2.85 & 1.25 & 33181 & -1.168 & .243 & \\
\hline
\end{tabular}

\section{Discusión}

El estudio analiza las actitudes hacia las personas refugiadas, considerando la percepción de amenaza que suponen y las reacciones afectivas que generan, y el grado de ciudadanía europea inclusiva que poseen los futuros maestros españoles, atendiendo su valoración sobre la Unión Europea y la cesión de derechos a las personas refugiadas. Asimismo, profundiza en la relación que se establece entre las actitudes hacia refugiados y la conformación de una ciudadanía europea abierta a la llegada de personas migrantes forzadas bajo el estatuto de ciudadanos de plenos derechos. Para lo cual se han adaptado y validado las escalas de amenaza y derechos y reacciones afectivas y se ha creado la escala de ciudadanía europea inclusiva.

El primero de los hallazgos sobre las actitudes hacia los refugiados registradas es una notable indiferencia generalizada y una baja implicación hacia la situación de las 
personas refugiadas por parte de los futuros docentes que se aprecia en la tendencia a puntuaciones centrales en la escala de amenaza y derechos (cercana a 3) y a una baja desviación de los valores medios. La comparativa de las medias en la escala de ciudadanía europea inclusiva de quienes se encuentran por encima y por debajo del percentil 50, muestra una escasa distancia en los valores, mostrando nuevamente un patrón de respuestas muy similar y centrado en todas las personas participantes. De igual modo, la escala de reacciones afectivas se encuentra ligeramente por debajo de 5 (sobre 10), manifestando una tendencia emocional neutral. El desinterés y desinformación hacia las personas refugiadas entre los jóvenes es un fenómeno que también ha sido registrado en Portugal (Padilla \& Golberg, 2017). Y coincide con las encuestas realizadas por el CIS en Europa con el Eurobarómetro por la cual tan solo el 7\% de la población española considera la cuestión de las migraciones una prioridad social y política mientras que la media europea está en un $22 \%$ (Eurobarómetro, 2018). Esta falta de preocupación ha tratado de explicarse en base a diferentes motivos: por un lado, en términos de dimensión de la situación, se subraya una recepción de personas refugiadas muy inferior a la recibida por países de Centroeuropa y una falta de experiencia en la gestión de refugiados. Las expectativas en cuanto a la recepción no se han cubierto, lo cual genera evitar una percepción de presión migratoria. Otro mecanismo explicativo de carácter más ideológico y mediático, es la ausencia (hasta el momento) de un discurso mediático organizado y de partidos xenófobos fuertes que puedan explicar la crisis económica y la precariedad asociada culpando a personas migrantes y fomentando un discurso de la competencia por los recursos. Otros factores culturales y religiosos, más latentes, son la existencia de un sector católico y una izquierda "cosmopolita” pro-inmigración más amplia que en otros países (Dixon et al., 2018), la persistencia de ciertos valores cristianos, no explícitos, que se traducen en formas más activas de solidaridad y de apoyo humanitario, reforzándose la empatía de determinados sectores. A lo que se suma una historia reciente de emigración española presente en el inconsciente colectivo y que define la identidad española.

En contraste, los estudios que se han realizado en otros países de Europa muestran un sentido de amenaza elevado, actitudes menos positivas hacia los refugiados, con importantes diferencias según sean los países de procedencia, y un discurso de la imposibilidad de acoger más personas en sus países (Dixon et al, 2018; Plener et al. 2017). En Turquía, los jóvenes y futuros docentes muestran visiones mucho menos conniventes y empáticas con las migraciones forzosas, con altos niveles de rechazo (Tarman \& Gürel, 2017; Yitmen, \& Verkuyten, 2018). Según los estudios contrastados, a medida que el tamaño de la población refugiada aumenta se aprecia un empeoramiento de las actitudes, contraviniendo la hipótesis del contacto.

Otro de los aspectos relevantes de la investigación es la identificación de un sentimiento europeísta inclusivo entre los futuros maestros. Esto vuelve a coincidir con los análisis del Eurobarómetro de 2018 en los que analizan que el $88 \%$ de la población española se considera europeísta, muy por encima de la mayoría de los Estados miembro. En este caso, no solo se materializa un reconocimiento con las instituciones y políticas europeas, sino que existe una posición favorable a la llegada de los refugiados y la concesión de derechos ciudadanos iguales. Entre los ítems actitudinales mejor puntuados se encuentra una oportunidad de construir una Europa más colectiva e inclusiva y percibiendo en su llegada una oportunidad para educar en convivencia. Al igual que sucedía con la percepción de amenaza, los derechos y las reacciones afectivas, esta actitud proclive a una Europa acogedora no es compartida en todos los territorios europeos. Observamos en nuestros estudiantes más europeísmo inclusivo que en otros países. Esta visión democrática de la Unión Europea entre los jóvenes españoles toma sus raíces en distintos 
mitos fundacionales. Uno es la consolidación de una España democrática con la entrada en la UE en 1986. Otro es la implementación de programas educativos de refuerzo identitario y de participación europea como los programas educativos supranacionales como el Erasmus (Cadavieco \& Pascual, 2013).

Las diferencias de género en la escala de ciudadanía, muestran una actitud más favorable por parte de las mujeres tanto en actitudes como en una ciudadanía inclusiva. Esta diferencia de género coincide con casi todos los estudios similares realizados. Los roles de género, el papel que asume la mujer en el ámbito reproductivo y de cuidados y la experiencia de desigualdad tiene un papel significativo en la construcción de una sensibilidad y una mayor empatía hacia el otro.

La cuarta idea fuerza que se deriva del estudio es la existencia de una relación entre actitudes hacia los refugiados y ciudadanía inclusiva. Cuanto menor es la actitud negativa cognitiva y emocional hacia los refugiados, más se defiende la acogida y cesión de derechos a los mismos desde Europa. La amenaza sentida es el factor que más predice la ciudadanía europea inclusiva, seguida de las reacciones afectivas positivas. Este aspecto pone de relieve la eficacia del uso de las teorías de la amenaza social para explicar el prejuicio a los inmigrantes (De Oliveira et al., 2005). Paradójicamente, la actitud favorable hacia los derechos públicos y sociales en refugiados (predisposición al acceso a la salud y educación) no tiene un carácter predictivo sobre la ciudadanía europea inclusiva. Esto puede explicarse por la existencia de una naturalización de derechos sociales que ya ofrecen cobertura a personas migrantes en el estado español.

No obstante, no se puede obviar que en España los estudios siguen analizando un racismo estructural que puede afectar en la integración real de los refugiados. Tal y como establecen Pareja y López (2018), la apropiada percepción y valoración hacia la acogida, o la ausencia de discriminación en abstracto no tiene por qué asociarse a una adecuada integración. Subrayan que, aunque los futuros docentes conceden el mismo valor a todas las culturas, sus posiciones difieren cuando se trata de clarificar el peso que la cultura mayoritaria debe jugar en la definición de la política a seguir, o la situación de desigualdad social que puedan experimentar los miembros de culturas distintas a la autóctona. Así, una valoración igualitaria no implica necesariamente una participación igualitaria y funcional en la organización de la vida social.

\section{Implicaciones para una educación intercultural $e$ inclusiva con refugiados para el profesorado}

La permanencia de una identidad europea inclusiva relativamente fuerte y la existencia de actitudes menos negativas hacia la inmigración que en otros países europeos no son suficientes para una integración efectiva del alumnado y sus familias refugiadas en los centros escolares. Es preciso desarrollar programas específicos dirigidos a propiciar la integración en las aulas, mejorar las actitudes de los docentes, las formas de estar y de relacionarse con la diversidad que convive, gestionar prácticas no discriminantes y democratizadoras en la escuela y adaptar transculturalmente los currículos (Banks, Suárez-Orozco, \& Ben-Peretz, 2016). Se trata de modificar los paradigmas socioeducativos que entienden al refugiado como una carga a la que hay que atender. En definitiva, programas que atiendan a las formas de ser, estar, de actuar y de saber de las y los docentes en el aula.

En base a los resultados obtenidos en este estudio, pueden esbozarse una serie de recomendaciones para futuros programas de formación educativa intercultural dirigidos al trabajo específico con personas refugiadas, que puedan ser implementados desde las propias universidades entendidas como esferas públicas democráticas (Giroux, 2016).

En primer lugar, un programa formativo de educación en contextos de refugio debe conocer las principales realidades migratorias, las causas de solicitud de refugio y asilo de las personas que migran forzosamente y las 
historias coloniales de los países que acogen. Comprender la corresponsabilidad de los países europeos en muchos de los contextos bélicos es una condición necesaria para generar un compromiso del profesorado con la atención y acogida a los menores y, de este modo, disminuir el desconocimiento y la indiferencia identificada. A su vez, el conocimiento de las particularidades migratorias, permitirá a los maestros en formación abordar de una manera más precisa y cuidadosa las situaciones que se les presenten según los países y culturas de procedencia.

En segundo lugar, y en respuesta al papel que muestra la amenaza social en la construcción y predicción de una ciudadanía inclusiva, hay que tratar de minimizar en los futuros docentes la percepción de peligro y cuestionamiento a su estatus como ciudadano y como docente. Uno de los recursos para trabajar la amenaza, acogiéndonos a su relación con las teorías de la identidad social, puede ser ejercitar la deconstrucción de identidades nacionales fuertes (identidades simples) y excluyentes y transitar hacia modelos identitarios más sanos, que transciendan el estado-nación, identidades de múltiple pertenencia, transculturales, más flexibles e inclusivas (identidades complejas) (Soriano, 2011). El trabajo sobre la identidad cultural del docente puede ligarse a un ejercicio de rastreo en las historias migratorias personales y colectivas, un trabajo donde la memoria histórica permita construir identidades mestizas, más empáticas con la migración como fenómeno humano universal. Esto favorecerá responder a uno de los retos que propone Banks et al. (2016) de la necesidad de integrar y cohesionar sin asimilar. Otra estrategia para disolver la percepción de amenaza real y simbólica implica el trabajo de prejuicios y rumores, siendo el factor predictor fundamental para una actitud europea acogedora. Desarticular el racismo revisando los privilegios que genera el orden social y racial en las personas no racializadas.

La importancia de las reacciones afectivas permite aventurar que desarrollar programas emocionales también pueda tener un efecto positivo en el grado de aceptación de las personas refugiadas, así como de las políticas europeas migratorias. La dimensión emocional hacia "los otros" percibidos como distintos a mí, suele ejercitarse mediante el trabajo de la empatía, es decir, la tarea de ubicarse en el lugar del otro para reconfigurar las reacciones que generan. El trabajo de la empatía como vía de modificación emocional exige que previamente se generen espacios de comunicación emocional individual e interpersonal, de manera que el futuro profesorado pueda experimentar un desarrollo.

Por último, con un sentido más estratégico, la formación debe generar climas escolares "clasroom climates" y ambientes escolares "scholar environment" en los que prime la diversidad (de procedencia, cultural, de "raza", de género, religiosas, de orientación sexual) y en los que se visibilice cómo se expresan las desigualdades y se cuestionen formas de multiculturalismo liberal, pudiendo recurrir a términos como el de superdiversidad y convivialidad (Castaño et al., 2011). Abordar las facetas estructurales racial colonial de las sociedades liberales es fundamental para generar prácticas educativas más justas.

\section{Conclusiones}

El estudio desarrollado hace una contribución al ámbito de las migraciones globales y la ciudadanía intercultural desde el terreno socioeducativo en el sureste español. Se reconocen algunas de las razones y de las fortalezas para iniciar programas formativos en educación intercultural y transcultural con el profesorado en formación aprovechando la cuestión de las personas refugiadas en un mundo globalizado.

La configuración de las actitudes y la construcción de una ciudadanía europea intercultural e inclusiva son particularmente sensibles a los cambios sociopolíticos, pudiendo sufrir rápidas modificaciones en el tiempo. Es por ello fundamental avanzar en 
propuestas formativas del profesorado y cambios curriculares que eviten actitudes excluyentes en nuestras escuelas.

\section{Referencias}

ACNUR (2016). Missing Out: Refugee Education in Crisis. Recuperado de: http://www.unhcr.org/missingout

Allport, G. (1954). The nature of prejudice. Reading, MA: Perseus Book Publishing.

Banks, J. A., Suárez-Orozco, M., \& BenPeretz, M. (Eds.). (2016). Global migration, diversity, and civic education: Improving policy and practice. Teachers College Press.

Cadavieco, J. F., \& Pascual, Á. (2013). Estudio de las acciones Erasmus en Educación Superior. Magister, 25(1), 74-82. doi: $\quad$ https://doi.org/10.1016/S02126796(13)70010-0

Castaño, F. J. G., Alcaraz, A. O., Gómez, M. R., \& Contini, P. (2011). Sobre multiculturalismos, críticas y superaciones conceptuales en la gestión de la diversidad cultural. In Inmigración, ciudadanía y gestión de la diversidad (pp. 31-65). Universidad Internacional de Andalucía.

De Lucas, J. (2001). Hacia una ciudadanía europea inclusiva. Su extensión a los inmigrantes. Revista CIDOB d'Afers Internacionals, 63-75.

De Oliveira, D. R., Techio, E. M., Páez, D., \& Herranz, K. (2005). Factores predictores de las actitudes ante la inmigración. Revista de psicología social, 20(1), 19-37. doi: https://doi.org/10.1174/0213474052871060

Dixon, T., Hawkins, S., Heijbroek, L., JuanTorres, M., \& Demoures, F. (2018). Attitudes towards ational Identity, Immigration and Refugees in Italy. London: More in Common Ltd.

Dryden-Peterson, S. (2016). Refugee education in countries of first asylum: Breaking open the black box of preresettlement experiences. Theory and Research in Education, 14(2), 131-148. doi: https://doi.org/10.1177/1477878515622703
García, M.C., Navas, M.S., Cuadrado, I. \& Molero, F. (2003). Inmigración y prejuicio: Actitudes de una muestra de adolescentes almerienses. Acción Psicológica, 2(2), 137147.

Giroux, H.A. (2016). La educación superior y las políticas de ruptura. Revista EntramadosEducación y Sociedad, 3(3), 15 - 26.

Gualda, E. (2011). Inmigración, ciudadanía y gestión de la diversidad. Universidad Internacional de Andalucía.

Li, G. (2018). Divergent paths, same destiny: A Bourdieusian perspective on refugee families' negotiation of urban school transition in the US. European Journal of $\begin{array}{llll}\text { Education. } & 0 & (0) . & \text { doi: }\end{array}$ https://doi.org/10.1111/ejed.12300

Liza G. Steele \& Lamis Abdelaaty (2018): Ethnic diversity and attitudes towards refugees, Journal of Ethnic and Migration Studies, doi: https://doi.org/10.1080/1369183X.2018.151 $\underline{3785}$

McWilliams, J. A., \& Bonet, S. W. (2016). Continuums of precarity: Refugee youth transitions in American high schools. International Journal of Lifelong Education, 35, 153-170. doi: https://doi.org/10.1080/02601370.2016.1164 $\underline{468}$

Murray, K. E., \& Marx, D. M. (2013). Attitudes toward unauthorized immigrants, authorized immigrants, and refugees. Cultural Diversity and Ethnic Minority Psychology, 19(3), $332 . \quad$ doi: https://doi.org/10.1037/a0030812

Padilla, B., \& Goldberg, A. (2017). Real and symbolic dimensions of the" refugee crisis" in Europe: a critical analysis from Portugal. REMHU: Revista Interdisciplinar da Mobilidade Humana, 25(51), 11-27.

Pareja, E. F. H., \& López, M. (2018). Interculturality and teacher education: A study from pre-service teachers' perspective. Australian Journal of Teacher Education. 43(3), 74. doi: https://doi.org/10.14221/ajte.2018v43n3.5 
Pettigrew, T. F. (1979). The ultimate attribution error: Extending Allport's cognitive analysis of prejudice. Personality and Social Psychology Bulletin, 5, 461-476.

Pettigrew, T.F. (1998). Intergroup contact theory. Annual Review of Psychology. 49(1), 65-85.

doi: https://doi.org/10.1146/annurev.psych.49.1.6 $\underline{5}$

Plener, P. L., Groschwitz, R. C., Brähler, E., Sukale, T., \& Fegert, J. M. (2017). Unaccompanied refugee minors in Germany: attitudes of the general population towards a vulnerable group. European child \& adolescent psychiatry, 26(6), 733-742. doi: $\quad$ https://doi.org/10.1007/s00787-0170943-9

Schweitzer, R., Perkoulidis, S.A., Krome, S. L.\& Ludlow, C. N. (2005) Attitudes towards Refugees: The Dark Side of Prejudice in Australia. Australian Journal of Psychology, 57(3), 170-179. doi: https://doi.org/10.1080/00049530500125199

Sharkey, J. (2018). The promising potential role of intercultural citizenship in preparing mainstream teachers for im/migrant populations. Language Teaching Research, 22(5), 570-589. doi: https://doi.org/10.1177/1362168817718577

Sidanius J. \& Pratto, F (1999). Social Dominance: An intergroup theory of social hierarchy and oppression. New York: Cambridge University Press. doi: https://doi.org/10.1017/CBO9781139175043

Soriano, E. (2011). Diagnóstico de la identidad cultural en escuelas multiculturales e intervención educativa. En E. Soriano (Coord) La interculturalidad como factor de calidad educativa (pp.175208). Madrid: La Muralla.

Stark, T. H., Mäs, M., \& Flache, A. (2015). Liking and disliking minority-group classmates: Explaining the mixed findings for the influence of ethnic classroom composition on interethnic attitudes. Social science research, 50, 164-176.
Stephan, W. G., \& Stephan, C. (1993). Cognition and affect in stereotyping: Parallel interactive networks. En D. M. Mackie, \& D. L. Hamilton (Eds.), Affect, cognition and stereotyping: Interactive processes in group perception (pp. 111 136). Orlando, FL: Academic Press. doi: https://doi.org/10.1016/B978-0-08-0885797.50010-7

Stephan, W. \& Stephan, C. (2000) An integrated threat theory of prejudice. In: Oskamp, S. (ed.) Reducing prejudice and discrimination. New Jersey: Laurence Erlbaum.

Stephan, W. G., Ybarra, O., \& Bachman, G. (1999). Prejudice Toward Immigrants. Journal of Applied Social Psychology, 29(11), 2221-2237. doi: https://doi.org/10.1111/j.15591816.1999.tb00107.x

Tajfel, H \& Turner, J. (1979). An integrative theory of intergroup conflict. En S. Worchel \& W. G. Austin (Eds). Psychology of intergroup relations (pp. 33-47). Chicago: Nelson-Hall Publishers.

Tarman, B., \& Gürel, D. (2017). Awareness of social studies teacher candidates on refugees in Turkey. The Journal of Social Studies Research, 41(3), 183-193. doi: https://doi.org/10.1016/j.jssr.2016.11.001

Wrench, A., Soong, H., Paige, K., \& Garrett, R. (2018). Building spaces of hope with refugee and migrant-background students. International Journal of Inclusive Education, 22(11), 1197-1212. doi: https://doi.org/10.1080/13603116.2017.1420 $\underline{251}$

Yitmen, Ş., \& Verkuyten, M. (2018). Positive and negative behavioural intentions towards refugees in Turkey: The roles of national identification, threat, and humanitarian concern. Journal of Community \& Applied Social Psychology. doi: https://doi.org/10.1002/casp.2354 


\begin{tabular}{l} 
Authors / Autores \\
\hline Verónica C. Cala (vcc284@ual.es) \\
Licenciada en Medicina, doctora en Educación para la Salud Transcultural. Es \\
la autora de contacto para este artículo. Miembro del grupo de investigación \\
”Investigación y evaluación en educación intercultural” (HUM-665) y \\
miembro del Centro de Estudio de las Migraciones y las Relaciones \\
Interculturales (CEMyRI) de la Universidad de Almería (España). Su \\
dirección postal es: Edificio Central, Planta 2, Despacho 11. 04120 - La \\
Cañada de San Urbano, Almería (España) \\
\hline Encarna Soriano-Ayala (esoriano@ual.es) \\
Profesora Titular del área de Métodos de Investigación y Diagnóstico en \\
Educación. Directora del grupo de investigación “Investigación y evaluación \\
en educación intercultural (HUM665) y miembro del Centro de Estudio de las \\
Migraciones y las Relaciones Interculturales (CEMyRI) de la Universidad de \\
Almería (España). Su dirección postal es: Edificio Central, Planta 2, \\
Despacho 12. 04120 - La Cañada de San Urbano, Almería (España). \\
\hline Manuel J. López-0625 \\
Profesor del Departamento de Educación, área de conocimiento de Didáctica \\
de las Ciencias Sociales. Es miembro del grupo de investigación \\
"Investigación y evaluación en educación intercultural (HUM665) Su \\
dirección postal es: Edificio Departamental de Humanidades y Ciencias de la \\
Educación I (Edif. A), Planta 2, Despacho 36. 04120 - La Cañada de San \\
Urbano, Almería (España). \\
\hline
\end{tabular}

\title{
RELIEVE
}

Revista ELectrónica de Investigación y EValuación Educativa E-Journal of Educational Research, Assessment and Evaluation

[ISSN: 1134-4032]$$
\text { (). }(1) \otimes
$$

Esta obra tiene licencia de Creative Commons Reconocimiento-NoComercial 4.0 Internacional. This work is under a Creative Commons Attribution 4.0 International license.
\end{abstract}

